Check for updates

Cite this: Chem. Sci., 2019, 10, 7023

๑ All publication charges for this article have been paid for by the Royal Society of Chemistry

Received 21st May 2019

Accepted 11th June 2019

DOI: $10.1039 / c 9 s c 02466 b$

rsc.li/chemical-science

\section{A nano-cocktail of an NIR-II emissive fluorophore and organoplatinum(ı) metallacycle for efficient cancer imaging and therapy $\dagger$}

\author{
Feng Ding, $\dot{t}^{\mathrm{a}}$ Zhao Chen, $\dot{\dagger}^{\mathrm{a}}$ Won Young Kim, $\dot{t}^{\mathrm{b}}$ Amit Sharma, ${ }^{\mathrm{b}}$ Chonglu $\mathrm{Li},{ }^{\mathrm{a}}$ \\ Qingying Ouyang, ${ }^{a}$ Hua Zhu, ${ }^{c}$ Guangfu Yang, ${ }^{a}$ Yao Sun (D) *a and Jong Seung Kim (D) *b
}

The scarcity of efficient imaging technologies for precise cancer treatment greatly drives the development of new nanotheranostic based platforms that enable both diagnostic and therapeutic functions, together in a single formulation. Owing to the complicated physiological microenvironment, nanosystems designed with the possibility of noninvasive real-time monitoring of therapeutic progression in the second near-infrared channel (NIR-II, 1000-1700 nm) could substantially improve the current cancer therapies. Herein, we design a novel NIR-II theranostic nanoprobe, PSY (size 110 $\mathrm{nm}$ ), by incorporating organoplatinum(॥) metallacycles P1 and an organic NIR-II molecular dye, SY1030, into the FDA-approved polymer Pluronic F127. Preliminary in vitro and in vivo studies suggest that PSY is capable of being internalized into glioma U87MG-cells with no significant internalization in non-cancerous tissues. In addition, it shows excellent photostability and minimal background for realtime monitoring the process of therapy in the NIR-II region. Furthermore, in U87MG xenografts and orthotopic breast tumor, PSY demonstrat significantly improved anticancer efficacy compared to a clinically approved Pt(॥)-based anticancer drug, cisplatin. The engineered nano-cocktail PSY offers a simple strategy for delivering the organoplatinum(II) macrocycle P1 and NIR-II fluorophore SY1030 as a cocktail of diagnostic and therapeutic functions and highlights its promising capacity for future cancer treatment.

\section{Introduction}

In the past decade, various types of supramolecular coordination complexes (SCCs) with finely tuned structures and sizes represented a promising platform for broad applications in many disciplines such as drug delivery, host-guest chemistry, and biosensors. ${ }^{1-3}$ Considering their potential application in biomedicine, a series of metal-based SCCs especially organoplatinum(II)-based ones have been recently actively explored to serve as cell imaging and antitumor agents owing to their promising properties such as great efficacy in inhibiting tumor growth and low toxicity for normal tissues compared with clinical Pt(II) drugs. ${ }^{4-9}$ Meanwhile, Pt(II)-based SCCs

${ }^{a}$ Key Laboratory of Pesticides and Chemical Biology, Ministry of Education, International Joint Research Center for Intelligent Biosensor Technology and Health, Center of Chemical Biology, College of Chemistry, Central China Normal University, Wuhan 430079, China. E-mail: sunyaogbasp@mail.ccnu.edu.cn

bDepartment of Chemistry, Korea University, Seoul 02841, Korea. E-mail: jongskim@ korea.ac.kr

'Key Laboratory of Carcinogenesis and Translational Research, Ministry of Education, Peking University Cancer Hospital \& Institute, Beijing 100142, China

$\dagger$ Electronic supplementary information (ESI) available. See DOI: $10.1039 / \mathrm{c} 9 \mathrm{sc} 02466 \mathrm{~b}$

\$ These authors contributed equally to this work. demonstrate interesting photo-physical properties including the ability to emit in the visible spectrum, allowing the delivery and release of organoplatinum(II) to be monitored at the cellular level. ${ }^{5,10-13}$ Despite these excellent properties, the potential decomposition of organoplatinum(II)-based SCCs in the presence of amino acids (histidine, lysine, arginine etc.) is the major obstacle for successful in vivo applications. ${ }^{14,15}$ Moreover, the poor photo-stability, low tumor accumulation and limited penetration depth of organoplatinum(II)-based SCCs in biological tissues remain additional challenges. ${ }^{15,16}$ Therefore, developing novel organoplatinum(II)-based SCC theranostic agents with longer emission wavelengths and having excellent photostability, high tumor uptake and efficacy in suppressing tumor growth is urgently demanded.

NIR-II imaging holds advantages of providing information on disease location and development due to deep penetration, low interfering signals and high signal to background ratio. ${ }^{17-23}$ Inspired by these excellent advantages of the NIR-II channel for in vivo bioimaging, diverse organic and inorganic emitters, such as rare earth materials, quantum dots, organic dyes, polymers, and carbon nanotubes, have been widely exploited in this novel fluorescence channel. ${ }^{24-36}$ Biological practices such as vascular/ lymphatic and tumor imaging with high contrast as well as precise image-guided surgery indeed bring good news for 
biomedical practices in the NIR-II channel. ${ }^{37-43}$ By combining chemotherapy with NIR-II imaging techniques to construct a theranostic platform for tumor therapy, tumors can be precisely located with strong NIR-II fluorescent signals. Additionally, owing to the stable NIR-II fluorescence, the tumor profile can be long-term clearly delineated to dynamically monitor tumor development, thus indirectly reflecting the efficiency of chemotherapy. There is no doubt that integrating this promising imaging modality with high-performance NIR-II probes will create a bright future for real-time image-guided therapeutic courses.

Herein, we designed a novel NIR-II nanotheranostic agent (PSY, Fig. 1) that comprised organoplatinum(II) metallacycles (P1) and an NIR-II molecular dye (SY1030) both incorporated into an FDA-approved amphiphilic polymer of poly(ethylene oxide)-poly(propylene oxide)-poly(ethylene oxide) (Pluronic F127), which was expected to present the following advantages. First, the incorporation of SY1030 into the PSY nanoprobe would achieve favorable photo-stability and bright fluorescence for biomedical applications. ${ }^{4-47}$ Second, owing to the emission of PSY shifting to the NIR-II channel (1.0-1.7 $\mu \mathrm{m})$, high-quality images of precise diagnosis and therapy monitoring in vivo could be obtained with deep tissue penetration and at micronscale resolution. ${ }^{4-50}$ Third, the utilization of F127 could not only endow PSY with excellent solubility and biocompatibility, ${ }^{51,52}$ but also enable higher preferential accumulation of both P1 and SY1030 at tumor sites. ${ }^{52}$ In contrast to the traditional Pt(II)-based drug cisplatin, our results demonstrated that the designed PSY nanoparticles exhibited a high level of tumor uptake with a superior signal to noise ratio $(\mathrm{S} / \mathrm{N})$ ratio for guiding therapy, enhancing the antitumor activities and lowering the adverse side effects in a U87MG tumor-bearing mice model.

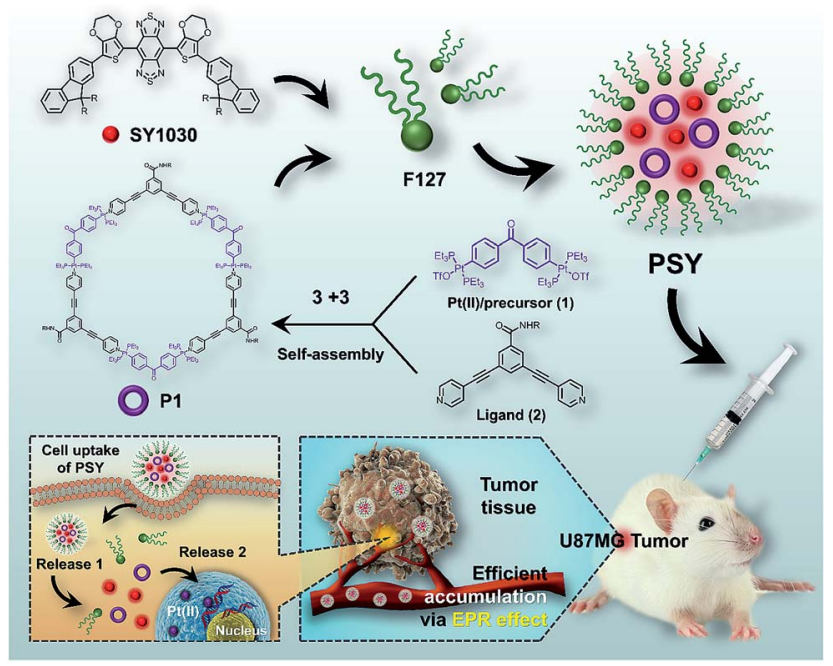

Fig. 1 The NIR-II molecular dye SY1030; the synthesis and selfassembly of the $\mathrm{Pt}(\mathrm{II})$ metallacycles $\mathrm{P} 1$, and the illustration of the cellular uptake of PSYand Pt(I) release from PSY.

\section{Results and discussion}

The procedure to prepare PSY was illustrated in Fig. 1. SY1030 was designed by using an optimized donor-acceptor-donor scaffold including the donor 3,4-ethylenedioxythiophene and the shielding unit dialkylfluorene, which contributed to an enhanced quantum yield (QY). ${ }^{53}$ SY1030 was synthesized through a convergent route in $10 \%$ overall yield in 9 steps (see the ESI $\dagger$ ) and characterized by ${ }^{1} \mathrm{H} /{ }^{13} \mathrm{C}-\mathrm{NMR}$ and MALDI-TOF-MS (Fig. S1-S3†). In the light of the principle of directional bonding, organoplatinum(II) metallacycles (P1) could be easily synthesized in high yield by combining a $60^{\circ} \mathrm{Pt}(\mathrm{II})$ precursor 1 and $120^{\circ}$ ligand 2 at a $1: 1$ molar ratio (Fig. 1 ). The conformation of P1 was confirmed by ${ }^{1} \mathrm{H}-\mathrm{NMR}$, multinuclear NMR and ESI-TOF-MS (Fig. S4-S6†). In the ${ }^{1} \mathrm{H}-\mathrm{NMR}$ spectrum, the protons of the pyridine $\mathrm{H}_{2 \mathrm{a}}$ and $\mathrm{H}_{2 \mathrm{~b}}$ in $\mathbf{P 1}$ were shifted downfield compared with those of the free ligand (2) due to the formation of the Pt-N bond (Fig. $\mathrm{S} 4 \dagger$ ). As shown in Fig. S5, $\uparrow$ P1 displayed an upfield shift of approximately $5.41 \mathrm{ppm}$ compared with the relevant ${ }^{31} \mathrm{P}\left\{{ }^{1} \mathrm{H}\right\}$ NMR spectra of the Pt(II) precursor 1 and also showed a sharp singlet peak at $c a$. 16.71 ppm corresponding to a single phosphorus environment. ESI-TOF-MS provided the evidence for the formation of the metallacycle structure of P1 with the loss of OTf anions $(m / z=1728.36,1258.67,1057.07$ and 789.35 , consistent with that for $[\mathrm{M}-3 \mathrm{OTf}]^{3+},[\mathrm{M}-4 \mathrm{OTf}]^{4+},[\mathrm{M}$ $-5 \mathrm{OTf}]^{5+}$ and $[\mathrm{M}-6 \mathrm{OTf}]^{6+}$ ).

PSY has been facilely synthesized via both P1 and SY1030 being encapsulated into F127 (2:1:7, w/w, Fig. 1 and ESI $\dagger) .{ }^{52}$ The fluorescence maximum absorption and emission wavelengths of PSY were observed at $\sim 750 \mathrm{~nm}$ and $\sim 1030 \mathrm{~nm}$, respectively, demonstrating a strong NIR-II fluorescence signal (Fig. 2a). Based on transmission electron microscopy (TEM) and dynamic light scattering (DLS), the as-synthesized PSY nanoparticles were discrete and uniform with an average size of $\sim 110 \mathrm{~nm}$ (Fig. 2b and c). The short- and long-term stability of PSY was tested in $37^{\circ} \mathrm{C}$ warm FBS, PBS and water solution by DLS measurement (Fig. 2d and S7 $\dagger$ ). Even after 7 days, there was no apparent change in particle-diameter distribution, suggesting excellent in vitro stability of PSY. Furthermore, in vitro release of Pt(II) from PSY (2.5 $\mathrm{mg} \mathrm{mL}^{-1}$ ) was studied using a dialysis bag diffusion method and quantified with inductively coupled plasma mass spectrometry (ICP-MS). The result elucidated that almost $\sim 50 \%$ of $\mathrm{Pt}(\mathrm{II})$ was released from PSY NPs due to the dissolution process of F127-based materials over $24 \mathrm{~h}$ (Fig. 2e).$^{54,55}$ PSY exhibited high photo-stability with negligible decay compared with the FDA-approved NIR-I theranostic agent indocyanine green (ICG) under continuous irradiation at $808 \mathrm{~nm}$ for $1 \mathrm{~h}$ (Fig. 2f). Also, PSY didn't show any significant change in fluorescence signal intensity upon irradiation in different media for a short- and long-term time (Fig. $2 \mathrm{~g}$ and S8 $\dagger$ ). The quantum yield (QY) of PSY in water was calculated as $\sim 0.08 \%$ using an IR-26 reference fluorophore $(\mathrm{QY}=0.05 \%) .{ }^{56,57}$ The amount of SY1030 encapsulated in PSY was also measured using ultraviolet-visible (UV-vis) spectrophotometer analysis. The SY1030 encapsulation efficiency of PSY was found to be 87.4 $\pm 1.7 \%$ (Fig. $2 \mathrm{~h}$ and i). 

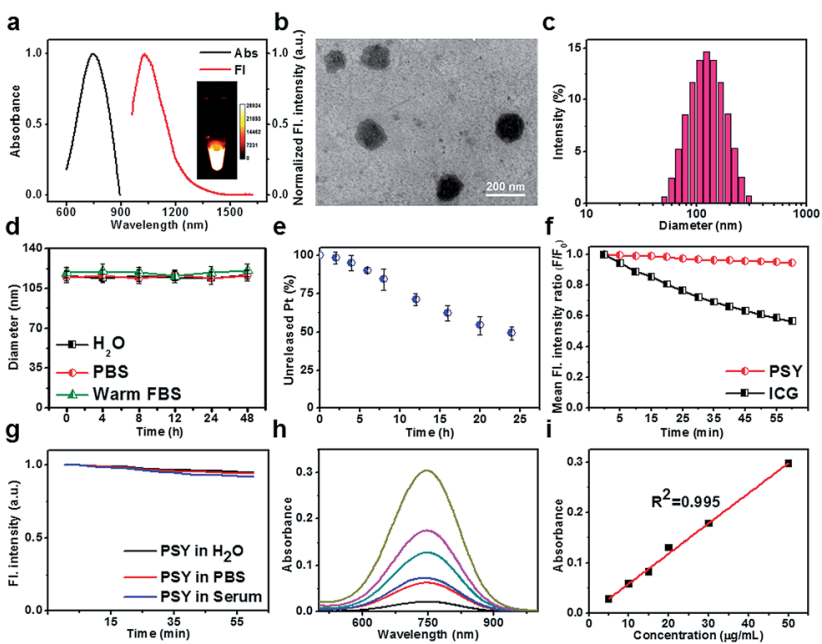

Fig. 2 Optical properties, size characterization and photo-stability of PSY in aqueous solution. (a) NIR-I absorbance and NIR-II fluorescence emission of PSY; (b) TEM image and (c) the DLS measurements of PSY; (d) in vitro stability of PSY in $37^{\circ} \mathrm{C}$ warm FBS, PBS and water solution after various incubation times ( $n=3$, the diameters of PSY with maximal intensity were determined by DLS measurements); (e) release of Pt from PSY at various times $(n=3)$; ( $f$ ) fluorescence stability of PSY and ICG (1.0 W cm $\left.\mathrm{W}^{-2}, 10 \mathrm{~ms}, 1000 \mathrm{LP}\right)$; (g) photo-stability of PSY in different media under continuous $808 \mathrm{~nm}$ irradiation $\left(1 \mathrm{~W} \mathrm{~cm}{ }^{-2}, 10\right.$ ms, 1000 LP); (h) UV-vis spectrum of PSY at different concentration; (i) calibration curve of PSY. The amount of SY1030 encapsulated in F127 was measured using a UV-vis spectrophotometer at $750 \mathrm{~nm}$. The calibration curve was linear in the range of $5-50 \mu \mathrm{g} \mathrm{mL} \mathrm{m}^{-1}$ with a correlation coefficient of $R^{2}=0.995$. The encapsulation efficiency was calculated as the ratio between the amount of SY1030 encapsulated in the F127. The organic dye SY1030 encapsulation efficiency of PSY was $87.4 \pm 1.7 \%$.

PSY was first investigated for its efficient cellular uptake behavior and imaging of the cancer cells. As shown in Fig. 3a and b, after incubation with PSY (containing 200 nM SY1030)

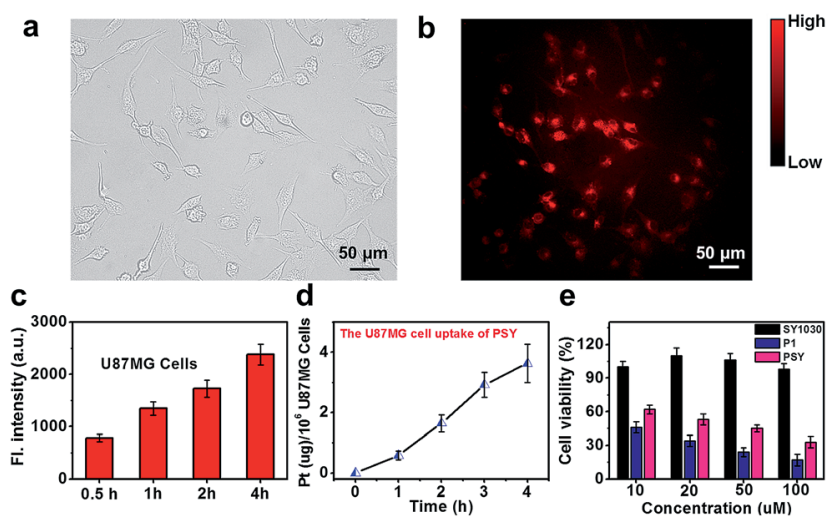

Fig. 3 Cellular uptake and relative viability of U87MG cells after incubation with PSY NPs. (a) Bright and (b) NIR-II fluorescence images of U87MG cells after incubation with PSY for $4 \mathrm{~h}$; (c) average NIR-II fluorescence of U87MG cells and the error bars were obtained by taking the s.d. of average fluorescence intensity from 20 cells in each NIR-II fluorescence image; (d) time-dependent quantitative analysis of the amount of platinum in the U87MG cells after incubation $(n=3)$; (e) cellular toxicity of SY1030, P1 and PSY on U87MG cells $(n=3)$. for $1 \mathrm{~h}$, the U87MG cells showed strong intracellular NIR-II fluorescence due to an efficient endocytosis pathway. The corresponding average fluorescence intensity from cells increased with extending the incubation time (Fig. 3c). The cellular uptake behavior and the amount of Pt released from PSY were further quantified using ICP-MS analysis. Results shown in Fig. 3d revealed that the cellular uptake of PSY gradually increased from $1 \mathrm{~h}$ to $4 \mathrm{~h}$. We also normalized the biodistribution of $\mathrm{Pt}$ (II) in PSY versus protein content following previous literature. ${ }^{58}$ As for the subcellular distribution, nearly $\sim 98.2 \%$ of $\mathrm{Pt}(\mathrm{II})$ content was within the nucleus, whereas a smaller proportion of the $\mathrm{Pt}(\mathrm{II})$ content was found in the cytoplasm (Table S1†). According to previous reports, this may be attributed to the fact that PSY was deconstructed at low $\mathrm{pH}$ in endo/lysosomes after internalization and the section including Pt(II) entered the nuclei and coordinated with the DNA. ${ }^{\mathbf{1 6 9 5 - 6 3}}$ The in vitro anticancer efficacy of SY1030, P1 and PSY (the concentration is calculated as Pt(II) content) was assessed using a 3-(4,5-dimethylthiazole-2-yl)-2,5diphenyl tetrazolium bromide (MTT) assay. As expected, the cell viabilities relied on the dosage of P1 and PSY that exhibited high anticancer activity toward U87MG cells (Fig. 3e). This may be attributed to their ability to form intra- and interstrand crosslinks on DNA. ${ }^{61,62}$ Above results provided convincing evidence for demonstrating the ability of PSY as a theranostic platform at the cellular level.

To investigate the in vivo pharmacokinetics of PSY and Pt(II) contents in the plasma at various time points, C57BL/6 mice were chosen. As shown in Fig. S9, $\dagger$ a strong fluorescent signal (NIR-II) was visualized from the liver region, suggesting that the clearance route of PSY was predominantly through the hepatobiliary system. Meanwhile, the result of ICP-MS indicated that PSY displayed a longer blood circulation time than the anticancer drug cisplatin, leading to enhanced efficacy over the traditional Pt(II) drug (Fig. 4a). Moreover, the phantom imaging in the NIR-II region indicated that improved imaging quality was obtained with SY1030 over a traditional NIR-I agent ICG (Fig. 4b). The advantages garnered by the brightest fluorescence of PSY in the NIR-II channel were clearly demonstrated in the following in vivo circulation network applications. First, the popliteal and sacral lymphatic nodes relevant to tumor metastasis were visualized in a dorsal angle in the NIR-II channel, demonstrating excellent $\mathrm{S} / \mathrm{N}$ of $\sim 18$ after intradermal administration of PSY at the rear paw of C57BL/6 mice (Fig. 4c and d). Second, PSY was capable of carrying out imaging and tracking of the vessel network in the C57BL/6 mice brain with high SBR as well as sharp images ( 19 and $169 \mu \mathrm{m}$, Fig. 4e and $\mathrm{f}$ ) in a noninvasive manner. Finally, the high-resolution fluorescent images of U87MG tumor blood vessels were also highlighted compared with the surrounding tissue after injection with PSY (Fig. 4g).

The global burden of the most common and aggressive brain tumor in adults, i.e., glioblastoma, is already enormous, and an exponential increase is predicted for the next couple of decades. ${ }^{64-68}$ Based on these challenges, U87MG tumor-bearing mice were established to assess the in vivo theranostic potential of PSY, and a clinically approved anticancer drug, cisplatin, was set as a positive control. The NIR-II fluorescence signal at the 
a

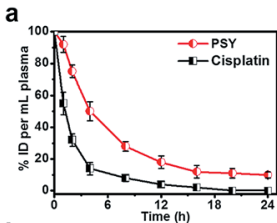

b

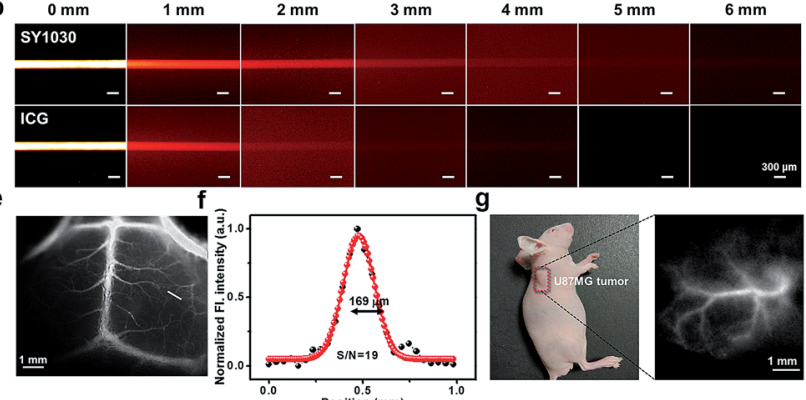

Fig. 4 In vivo representative images with PSY. (a) In vivo blood elimination kinetics of cisplatin and PSY at a dose of $2 \mathrm{mg}$ Pt per kilogram body weight ( $n=3$ for each group); (b) NIR-II fluorescence images of a capillary tube filled with PSY and ICG solution immersed at different depths in 1\% Intralipid ( $82 \mathrm{~mW} \mathrm{~cm}^{-2}, 150 \mathrm{~ms}$, LP 1000); (c-g) NIR-II images of lymphatic vessels, brain vessels, and U87MG tumor blood vessels after injection of PSY with the width and SBR, respectively, under $808 \mathrm{~nm}$ laser illumination $\left(82 \mathrm{~mW} \mathrm{~cm}^{-2}, 100 \mathrm{~ms}, 1000\right.$ LP).

U87MG tumor region was observed clearly within $24 \mathrm{~h}$ postinjection (Fig. S10†). Besides, an ex vivo biodistribution of PSY in tumors and organs indicated high accumulation in the tumors, liver and spleen (Fig. S11 $\dagger$ ). Further, the tissue distribution of Pt(II) content from PSY or cisplatin was also evaluated quantitatively. Compared with free cisplatin, significant accumulation of Pt(II) of PSY in the tumor region was obviously observed, which was attributed to the passive targeting (Fig. S12 $\dagger$ ). More importantly, in the case of PSY a lower Pt(II) uptake by normal organs was observed, suggesting that PSY could decrease the systemic toxicity of Pt-based drugs to normal tissues and organs resulting in minimal associated side-effects and improved overall efficacy.

To assess the anticancer efficacy, U87MG-inoculated mice were intravenously injected with PSY, free cisplatin as a positive control (dose: $2 \mathrm{mg}_{\mathrm{Pt}} \mathrm{kg}^{-1}$ body weight) and SY1030/F127 and PBS as negative controls. As shown in Fig. 5a, the fluorescence signal from the localized PSY at the tumor region could be dynamically monitored during the whole process of therapy thanks to the excellent photo-properties of PSY. To evaluate the adverse side effects of the above formulations, body weights of mouse models after treatment were recorded during the experimental period and the major organs of the sacrificed groups were analyzed on the last day with hematoxylin-eosin staining (H\&E). Serious body weight loss was observed in the cisplatin-treated group (Fig. 5b). Also, from H\&E analysis result, it was observed that the primary organs from cisplatin-treated mice were severely damaged (Fig. 5c). However, the PSYtreated group just as SY1030/F127 and PBS-treated groups did not exhibit any obvious body weight loss and organs damage, indicating better in vivo biocompatibility and negligible systemic toxicity (Fig. $5 \mathrm{~b}$ and c). Moreover, tumor size of mice at



Fig. 5 In vivo therapeutic efficacy of PSY NPS. (a) NIR-II images of monitoring the PSY therapeutic response in U87MG tumors $182 \mathrm{~mW}$ $\mathrm{cm}^{-2}, 100 \mathrm{~ms}$, LP 1000); (b) average body weights were analyzed; (c) H\&E stained images of the heart, liver, spleen, lung and kidney from different groups after intravenous injection of PBS, SY1030/F127, cisplatin or PSY. Scale bar: $100 \mu \mathrm{m}$; (d) U87MG tumor growth inhibition curves for PBS, cisplatin, SY1030 and PSY; (e) U87MG tumor weight; (f) survival rate of the mice bearing U87MG tumors after different treatments.

every $3 \mathrm{~d}$ and the weight of tumors taken out at the last day were measured to observe the efficacy of PSY in tumor inhibition. As demonstrated in Fig. 5d and e, the SY1030/F127-treated and PBS-treated groups showed an obvious tumor increase. Although treatment with cisplatin achieved moderate tumor growth inhibition, PSY exhibited the highest tumor inhibition efficiency. It could be attributed to the high accumulation of Pt(II) from PSY at tumor sites compared with traditional cisplatin treatment. The Kaplan-Meier survival plots are shown in Fig. $5 \mathrm{f}$. Moreover, the mice in the established U87MG tumor model group treated with cisplatin were not able to survive after $46 \mathrm{~d}$ due to the severe damage of the primary organs by the free Pt(II) drug. In contrast, PSY with lower systemic toxicity and efficient tumor inhibition significantly prolonged the overall survival of the mice in the tested group (Fig. 5f).

Furthermore, immunohistochemical examination of tumors excised from mice treated with different formulations was 
performed to assess the therapeutic efficacy of PSY. As demonstrated in Fig. S13, $\uparrow$ for H\&E staining, the tumor tissues of PSY-treated group displayed the highest death of tumor cells, indicating that PSY has high antitumor activity. Subsequently, Ki67-positive immunohistochemical staining further proved significantly inhibited cell proliferation capability in the PSY-treated group. Moreover, TdT-mediated dUTP-biotin nick and labeling (TUNEL) staining also confirmed that the number of apoptotic tumor cells in the PSY-treated group was the highest. These results obviously were in accordance with the enhanced therapeutic outcome of PSY in in vivo anticancer experiments.

Further, to verify the universal anticancer efficacy of PSY and display the possibility of translating it into further clinical development, an orthotopic breast tumor model was chosen $(n$ $=3$ ). Similar to therapeutic experiments on the U87MG tumorbearing model, free cisplatin (dose: $2 \mathrm{mg}_{\mathrm{Pt}} \mathrm{kg}^{-1}$ body weight), SY1030/F127 and PBS were set as controls. Body weight and tumor volume were recorded every 2 d. As shown in Fig. S14a, $\uparrow$ a high NIR-II fluorescent signal intensity was observed in the breast tumor region, indicating the good accumulation of PSY in the tumor. The whole process of therapy for $15 \mathrm{~d}$ could be also monitored with the help of PSY. PSY-treated mice showed an ascending body weight, in contrast, cisplatin displayed a significant decrease of body weight, indicating good biocompatibility and non-obvious systemic toxicity of PSY (Fig. S14b $\dagger$ ). Besides, as demonstrated in Fig. S14c and d, $\dagger$ the inhibition of tumor growth in the PSY treated group was more significant than in other groups.

\section{Conclusions}

In summary, a novel Pt(II)-based theranostic, PSY, that incorporated both organoplatinum(II) metallacycles and an NIR-II based organic dye into F127 was constructed, which could improve the distribution of $\mathrm{Pt}(\mathrm{II})$ in tumors compared with free cisplatin in the U87MG glioblastoma cancer model mice. PSY possessed good optical properties, such as decent photo-stability and bright fluorescence in the NIR-II region to visualize the blood circulation system and precisely locate the tumor sites with high resolution. Additionally, PSY accumulated at the tumor region preferentially. The efficient U87MG and orthotopic breast tumor inhibition rate with minimal adverse side effects prolonged the life span of tumor-bearing mice in the PSY-treated group. The cocktail based on the PSY platform clearly demonstrated its potential as a multifunctional platform. Besides, as recent reports proved that photoacoustic imaging (PAI) displays deeper tissue penetration than fluorescent imaging in the NIR-II region, ${ }^{69}$ a promising future can also be anticipated with this composite nano-platform for various PAI modality guided therapies.

\section{Conflicts of interest}

There are no conflicts to declare.

\section{Acknowledgements}

This work was partially supported by grants from the National Key R\&D Program (2017YFA0505203), NSFC (21708012), 111 project B17019, NSFHP (2017CFB151), financially supported by self-determined research funds of CCNU from the colleges, basic research and operation of MOE, Wuhan Morning Light Plan of Youth Science and Technology (201705304010321), the Open Research Fund of Key Laboratory of Carcinogenesis and Translational Research (Ministry of Education/Beijing) and CRI project (Grant No. 2018R1A3B1052702, J. S. K.). The animal experiments in NIR-II imaging studies were done in the Center for Animal Experiment of Central China Normal University (Wuhan, Hubei, P. R. China), which has been accredited by the Association for Assessment and Accreditation of Laboratory Animal Care International (AAALAC International), and all subsequent procedures were approved by and performed in accordance with the Guidelines for the Care and Use of Laboratory Animals of the Chinese Animal Welfare Committee.

\section{Notes and references}

1 T. R. Cook and P. J. Stang, Chem. Rev., 2015, 115, 7001.

2 G. Yu, K. Jieand and F. Huang, Chem. Rev., 2015, 115, 7240.

3 W. Wang, Y. Wang and H. Yang, Chem. Soc. Rev., 2016, 45, 2656.

4 W. Wang, Y. Zhang, B. Sun, L. Chen, X. Xu, M. Wang, X. Li, Y. Yu, W. Jiang and H. Yang, Chem. Sci., 2014, 5, 4554.

5 M. Zhang, S. Yin, J. Zhang, Z. Zhou, M. L. Saha, C. Lu and P. J. Stang, Proc. Natl. Acad. Sci. U. S. A., 2017, 114, 3044.

6 Y. Sun, F. Ding, Z. Zhou, C. Li, M. Pu, Y. Xu, Y. Zhan, X. Lu, H. Li, G. Yang, Y. Sun and P. J. Stang, Proc. Natl. Acad. Sci. U. S. A., 2019, 116, 1968.

7 C. Huang, L. Xu, J. Zhu, Y. Wang, B. Sun, X. Li and H. Yang, J. Am. Chem. Soc., 2017, 139, 9459.

8 T. C. Johnstone, K. Suntharalingam and S. J. Lippard, Chem. Rev., 2016, 116, 3436.

9 H. Yu, M. Li, G. Liu, J. Geng, J. Wang, J. Ren, C. Zhao and X. Qu, Chem. Sci., 2012, 3, 3145.

10 M. Yamashina, M. Sartin, Y. Sei, M. Akita, S. Takeuchi, T. Tahara and M. Yoshizawa, J. Am. Chem. Soc., 2015, 137, 9266.

11 G. Yin, H. Wang, X. Wang, B. Song, L. Chen, L. Wang, X. Hao, H. Yang and X. Li, Nat. Commun., 2018, 9, 567.

12 T. C. Johnstone, K. Suntharalingam and S. J. Lippard, Chem. Rev., 2016, 116, 3436.

13 J. L. Tsai, T. Zou, J. Liu, T. Chen, A. O. Chan, C. Yang, C. N. Lok and C. M. Che, Chem. Sci., 2015, 6, 3823.

14 M. Saha, X. Yan and P. J. Stang, Acc. Chem. Res., 2016, 49, 2527.

15 A. Gasini, B. Woods and M. Wenzel, Inorg. Chem., 2017, 56, 14715.

16 G. Yu, T. Cook, Y. Li, Z. Yan, D. Wu, J. Shao, J. Shen, G. Tang, F. Huang, X. Chen and P. J. Stang, Proc. Natl. Acad. Sci. U. S. A., 2016, 113, 13720.

17 G. Hong, A. L. Antaris and H. Dai, Nat. Biomed. Eng., 2017, 1, 0010 . 
18 H. Wan, J. Yue, S. Zhu, T. Uno, X. Zhang, Q. Yang, K. Yu, G. Hong, J. Wang, L. Li, Z. Ma, H. Gao, Y. Zhong, J. Su, A. L. Antaris, Y. Xia, J. Luo, Y. Liang and H. Dai, Nat. Commun., 2018, 9, 1171.

19 S. He, J. Song, J. Qu and Z. Cheng, Chem. Soc. Rev., 2018, 47, 4258.

20 Q. Chen, J. Chen, Z. Yang, Z. Dong and Z. Liu, Nano Res., 2018, 11, 5657.

21 Y. Cai, Z. Wei, C. Song, C. Tang, W. Han and X. Dong, Chem. Soc. Rev., 2019, 48, 22.

22 Q. Miao and K. Pu, Adv. Mater., 2018, 30, 1801778.

23 Kenry, Y. Duan and B. Liu, Adv. Mater., 2018, 30, 1802394.

24 Q. Yang, Z. Ma, H. Wang, B. Zhou, S. Zhu, Y. Zhong, J. Wang, H. Wan, A. Antaris, R. Ma, X. Zhang, J. Yang, X. Zhang, H. Sun, W. Liu, Y. Liang and H. Dai, Adv. Mater., 2017, 29, 1605497.

25 Q. Miao, C. Xie, X. Zhen, Y. Lyu, H. Duan, X. Liu, J. V. Jokerst and K. Pu, Nat. Biotechnol., 2017, 35, 1102.

26 F. Ding, Y. Zhan, X. Lu and Y. Sun, Chem. Sci., 2018, 9, 4370. 27 J. Qi, C. Sun, A. Zebibula, H. Zhang, R. T. Kwok, X. Zhao, W. Xi, J. W. Y. Lam, J. Qian and B. Tang, Adv. Mater., 2018, 30, 1706856.

28 Y. Fan, P. Wang, Y. Lu, R. Wang, L. Zhou, X. Zheng, X. Li, J. A. Piper and F. Zhang, Nat. Nanotechnol., 2018, 13, 941.

29 S. Zhu, S. Herraiz, J. Yue, M. Zhang, H. Wan, Q. Yang, Z. Ma, Y. Wang, J. He, A. L. Antaris, Y. Zhong, S. Diao, Y. Feng, Y. Zhou, K. Yu, G. Hong, Y. Liang, A. J. Hsueh and H. Dai, Adv. Mater., 2018, 30, 1705799.

30 O. T. Bruns, et al., Nat. Biomed. Eng., 2012, 1, 0056.

31 A. L. Antaris, et al., Nat. Mater., 2016, 15, 235.

32 Y. Jiang, P. K. Upputuri, C. Xie, Y. Lyu, L. Zhang, Q. Xiong, M. Pramanik and K. Pu, Nano Lett., 2017, 17, 4964.

33 C. Li and Q. Wang, ACS Nano, 2018, 12, 9654.

34 L. Liu, S. Wang, B. Zhao, P. Pei, Y. Fan, X. Li and F. Zhang, Angew. Chem., Int. Ed., 2018, 57, 7518.

35 G. Hong, J. C. Lee, J. T. Robinson, U. Raaz, L. Xie, N. F. Huang, J. P. Cooke and H. J. Dai, Nat. Med., 2012, 18, 1841.

36 H. He, Y. Lin, Z. Tian, D. Zhu, Z. Zhang and D. Pang, Small, 2018, 14, 1703296.

37 J. Jiang, et al., Adv. Mater., 2018, 30, 1705980.

38 S. Zhu, B. C. Yung, S. Chandra, G. Niu, A. L. Antaris and X. Chen, Theranostics, 2018, 8, 4141.

39 G. Xu, et al., Angew. Chem., Int. Ed., 2018, 130, 3688.

40 B. Li, L. Liu, M. Zhao, Z. Lei and F. Zhang, Angew. Chem., Int. Ed., 2018, 57, 7483.

41 Y. Tang, et al., Adv. Mater., 2018, 30, 1870226.
42 Y. Sun, et al., Chem. Sci., 2017, 8, 3489.

43 Y. Sun, et al., Chem. Sci., 2018, 9, 2092.

44 R. Wang, L. Zhou, W. Wang, X. Li and F. Zhang, Nat. Commun., 2017, 8, 14702.

45 E. D. Cosco, et al., Angew. Chem., Int. Ed., 2017, 56, 13126.

46 Z. Sheng, et al., Adv. Mater., 2018, 30, 1870214.

47 Z. Xue, S. Zeng and J. Hao, Biomaterials, 2018, 171, 153.

48 Q. Yang, et al., J. Am. Chem. Soc., 2018, 140, 1715.

49 X. Hao, C. Li, Y. Zhang, H. Wang, G. Chen, M. Wang and Q. Wang, Adv. Mater., 2018, e1804437.

50 B. Guo, Z. Sheng, D. Hu, C. Liu, H. Zheng and B. Liu, Adv. Mater., 2018, 30, 1802591.

51 O. S. Wolfbeis, Chem. Soc. Rev., 2015, 44, 4743.

52 J. Qi, C. W. Sun, A. Zebibula, H. Zhang, R. T. K. Kwok, X. Zhao, W. Xi, J. W. Y. Lam, J. Qian and B. Tang, Adv. Mater., 2018, 30, 1706856.

53 B. Zhou, Z. Hu, Y. Jiang, C. Zhong, Z. Sun and H. Sun, Phys. Chem. Chem. Phys., 2018, 20, 19759.

54 J. C. Gilbert, J. Hadgraft, A. Bye and L. G. Brookes, Int. J. Pharm., 1986, 32, 223.

55 A. Oshiro, et al., Langmuir, 2014, 30, 13689.

56 Q. E. Semonin, J. C. Johnson, J. M. Luther, A. G. Midgett, A. J. Nozik and M. C. Beard, J. Phys. Chem. Lett., 2010, 1, 2445.

57 F. Ding, C. Li, Y. Xu, J. Li, H. Li, G. Yang and Y. Sun, Adv. Healthcare Mater., 2018, 7, 1800973.

58 A. Naik, R. Rubbiani, G. Gasser and B. Spingler, Angew. Chem., Int. Ed., 2014, 53, 6938.

59 G. Yu, M. Zhang, M. L. Saha, Z. Mao, J. Chen, Y. Yao, Z. Zhou, Y. Liu, C. Gao, F. Huang, X. Chen and P. J. Stang, J. Am. Chem. Soc., 2017, 139, 15940-15949.

60 I. V. Grishagin, et al., Proc. Natl. Acad. Sci. U. S. A., 2014, 111, 18448-18453.

61 J. J. Wilson and S. L. Lippard, Chem. Rev., 2014, 114, 4470.

62 J. S. Butler and P. J. Sadler, Curr. Opin. Chem. Biol., 2013, 17, 175.

63 N. P. Farrell, Chem. Soc. Rev., 2015, 44, 8773.

64 N. Graf and S. J. Lippard, Adv. Drug Delivery Rev., 2012, 64, 993.

65 X. Wang and Z. Guo, Chem. Soc. Rev., 2013, 42, 202.

66 J. P. Almeida, K. L. Chaichana, J. Rincon-Torroella and A. Quinones-Hinojosa, Curr. Neurol. Neurosci. Rep., 2015, 15, 517.

67 F. Ding, S. Chen, W. Zhang, Y. Tu and Y. Sun, Bioorg. Med. Chem., 2017, 25, 5179.

68 F. Ding, Y. Fan, Y. Sun and F. Zhang, Adv. Healthcare Mater., 2019, DOI: 10.1002/adhm.201900260.

69 J. Li and K. Pu, Chem. Soc. Rev., 2019, 48, 38. 